\title{
Automated detection of micro-organisms in blood cultures by means of the Malthus Microbiological Growth Analyser
}

\author{
DFJ BROWN, M WARNER, CED TAYLOR, RE WARREN \\ From the Clinical Microbiology and Public Health Laboratory, Level 6, Addenbrooke's Hospital, Hills \\ Road, Cambridge
}

SUmmary A prototype Malthus Microbiological Growth Analyser was compared with conventional methods for examining blood cultures in a trial of 651 cultures mostly from patients with haematological malignancy or undergoing haemodialysis or renal transplantation. Of 100 significantly positive cultures, organisms from 82 grew in the conventional aerobic $\left(+\mathrm{CO}_{2}\right)$ bottle, 78 in the conventional anaerobic bottle and 71 in the Malthus bottle. The differences were not statistically significant $(\mathrm{p}>0.05)$. The Malthus system detected $83.6 \%$ of significantly positive cultures earlier than the comparable conventional bottles while $7 \cdot 3 \%$ positive cultures were detected earlier by the conventional system. When use of the Malthus system was restricted to the hours of 09.00 to 17.30 daily $27.3 \%$ positive cultures were detected earlier by the Malthus system and $16.4 \%$ were detected earlier by the conventional system. One of the organisms which grew in the Malthus bottle, a contaminating Staphylococcus epidermidis, was not detected by the Malthus system. Instability of electrodes resulted in $26.9 \%$ false positive cultures with the prototype Malthus system. Contamination rates in both the Malthus and conventional anaerobic bottles were lower than in the aerobic bottles.

Automated screening of blood cultures is desirable because conventional methods of visual examination and blind subculture are time-consuming, tedious, expensive in materials and labour, and run the risk of introducing contaminants during subculture. Automation might also benefit patients if the system were capable of earlier detection of microbial growth. Such an opportunity for earlier identification and antimicrobial susceptibility testing of infecting organisms might result in earlier diagnosis or more appropriate therapy of what are often life-threatening infections. There might also be secondary cost benefits associated with earlier and more appropriate diagnostic investigations and antimicrobial therapy.

Interest in detecting bacterial growth by monitoring electrical impedance of bacterial cultures has been revived with recent interest in rapid methods and automation in microbiology. ${ }^{12} \mathrm{~A}$ system of screening blood cultures based on measuring electrical impedance, the Bactometer, has been investigated. $^{34}$ Parallel studies of conventional and impedance-based methods of detection showed that

Accepted for publication 5 October 1983 earlier detection of positive cultures was possible by the impedance method but one report ${ }^{3}$ suggested that false-negative results were not uncommon. The Malthus system ${ }^{5}$ measures electrical conductivity rather than impedance. We have investigated a prototype version of the apparatus in a study of blood cultures examined in parallel by the Malthus system and by a conventional system.

\section{Material and methods}

BLOOD CULTURES

Six hundred and fifty-one blood cultures were received, mostly from patients with haematological malignancy, on haemodialysis or following renal transplantation. Clinicians were requested to take 45 $\mathrm{ml}$ blood when possible and to distribute it equally among three bottles each containing $85 \mathrm{ml}$ medium. One bottle was a Malthus cell which has an electrode integral with the cap and a septum for injecting blood. The other two were $120 \mathrm{ml}$ medical flat bottles. All three bottles in the set were returned to the laboratory where the Malthus bottle was separated from the conventional bottles. Examination of 
cultures by the conventional and Malthus methods was done independently by different staff in different parts of the laboratory.

\section{MEDIA}

The basal medium in all bottles was Brain Heart Infusion Broth (Difco) with supplements prepared as described by Holdeman et al. ${ }^{6}$ The bottles were not gassed with $\mathrm{CO}_{2}$. Before they were autoclaved $0.025 \% \mathrm{wt} / \mathrm{vol}$ sodium polyanethol sulfonate (Sigma) and $0.01 \% \mathrm{wt} / \mathrm{vol}$ para amino benzoic acid (Sigma) were added. After autoclaving, 50 units sterile broad spectrum beta-lactamase (Whatman) were injected into each bottle and $0.025 \% \mathrm{wt} / \mathrm{vol}$ sterile saponin (Sigma) was injected into the Malthus bottles only.

Blood agar plates consisted of a basal layer (Blood Agar Base No 2, Oxoid) and an overlay containing $4 \% \mathrm{wt} / \mathrm{vol}$ Heart Infusion Agar (Difco), $0.5 \% \mathrm{wt} / \mathrm{vol}$ Agar No 2 (Lab M) and 10\% vol/vol defibrinated horse blood (Difco). Cysteine, lactose, electrolytedeficient agar (CLED agar) was prepared as directed by the manufacturer (Oxoid). Neomycin blood agar contained $44 \mathrm{~g} / 1$ Columbia agar (Difco), $5 \mathrm{~g} / 1$ yeast extract (Difco), $500 \mathrm{mg} / \mathrm{l}$ cysteine hydrochloride (Sigma), $100 \mathrm{mg} / \mathrm{l}$ neomycin sulphate (Biorex Laboratories), $0.1 \mathrm{mg} / \mathrm{l}$ vitamin $\mathrm{K}_{3}$ (Sigma), $2.5 \%$ vol/vol defibrinated horse blood (Difco) lysed with saponin and $7.5 \%$ (vol/vol) defibrinated horse blood (Difco).

CONVENTIONAL BLOOD CULTURES

Bottles were incubated at $37^{\circ} \mathrm{C}$, the aerobic bottles with their caps loose in a $\mathrm{CO}_{2}$ incubator and the anaerobic bottles with their caps tight. All bottles were examined for turbidity twice daily for six days. Aerobic bottles were routinely subcultured after incubating for one day, as were both bottles after incubating for three days and for nine days. On each occasion subcultures were made on to two plates of blood agar, one of each pair being incubated at $37^{\circ} \mathrm{C}$ in a $\mathrm{CO}_{2}$ incubator $\left(5 \% \mathrm{CO}_{2}\right)$ and the other at $37^{\circ} \mathrm{C}$ anaerobically $\left(90 \%\right.$ hydrogen, $\left.10 \% \quad \mathrm{CO}_{2}\right)$. Plates were examined after incubating for $24 \mathrm{~h}$ and again after $48 \mathrm{~h}$ if growth was not observed at $24 \mathrm{~h}$. In addition, the first day subculture incubated in a $\mathrm{CO}_{2}$ incubator was examined on the afternoon of the day of subculture.

When turbidity of the medium suggested bacterial growth, anaerobic and aerobic bottles were subcultured (anaerobic with a syringe and needle; aerobic with a disposable plastic loop) on to two plates of blood agar, one being incubated at $37^{\circ} \mathrm{C}$ in a $\mathrm{CO}_{2}$ incubator and the other anaerobically, as well as on to CLED agar incubated at $37^{\circ} \mathrm{C}$ in a $\mathrm{CO}_{2}$ incubator. If examination of Gram-stained smears of the medium revealed Gram-negative bacilli, the sample was subcultured on to neomycin blood agar also.
These cultures were incubated anaerobically at $37^{\circ} \mathrm{C}$. Subcultures were examined after incubating for $24 \mathrm{~h}$ and again after $48 \mathrm{~h}$ if growth was not observed at 24 h. Organisms isolated were identified by standard methods $^{7}$ except for Enterobacteriaceae and pseudomonads, which were identified by the use of API 20 E kits (API Laboratory Products).

\section{MALTHUS BLOOD CULTURES}

The prototype equipment used was the "Malthus 112L Microbiological Growth Analyser" (Malthus Instruments Ltd). It consisted of four waterbaths, each of which held 28 Malthus blood culture bottles. Each bottle contained a pair of platinum electrodes deposited on a ceramic strip, which was fixed into the moulded cap of the bottle. On receipt of inoculated blood culture bottles the electrodes were connected to the apparatus by electrical leads having push-on connectors. The electrical conductivity of the medium in the bottles was automatically scanned every $30 \mathrm{~min}$ for nine days and the data thereby obtained stored in a Motorola Exorset microcomputer. Data for individual bottles were available on demand either as a graph indicating change of conductivity with time on a visual display unit or on a digital plotter, or expressed numerically as change of conductivity on a visual display unit or matrix printer. Data were examined twice daily and any bottle showing accelerating changes in conductivity was subcultured (without disconnecting the bottle) by the use of a syringe and a $127 \mathrm{~mm} 16$ gauge stainless steel hypodermic needle (Coopers Needle Works Ltd). The sample was examined in a manner similar to that described above in the conventional system for bottles with turbidity that suggested bacterial growth.

If, out of ordinary laboratory working hours, a bottle could not be connected promptly to the Malthus system after inoculation with a blood sample it was possible for the culture to be fully grown before the bottle could be set up on the system. Consequently there was often no change in conductivity indicating bacterial growth. In an attempt to prevent this happening, bottles delivered to the laboratory and placed in an incubator during the early evening were connected to the Malthus apparatus at approximately 22.30 hours by the member of laboratory staff on-call. Any cultures taken later during the night were incubated and then subcultured the following morning before being connected to the apparatus.

After nine days, all Malthus bottles were subcultured as described for conventional bottles.

\section{Results}

POSITIVE BOTTLES

From 100 of 651 cultures, organisms considered to be 
Table 1 Numbers of blood cultures positive in the various bottles (out of 651 cultures)

\begin{tabular}{lcc}
\hline Bottles positive & \multicolumn{2}{c}{ Positive cultures } \\
\cline { 2 - 3 } & Significant & $\begin{array}{l}\text { Contaminant } \\
\text { only }\end{array}$ \\
\hline All & 60 & 5 \\
Aerobic only & 13 & 22 \\
Anaerobic only & 10 & 9 \\
Malthus only & 6 & 8 \\
Aerobic and anaerobic & 6 & 4 \\
Aerobic and Malthus & 3 & 1 \\
Anaerobic and Malthus & 2 & 2 \\
Total & 100 & 51 \\
\hline
\end{tabular}

Table 2 Organisms isolated from various bottles

\begin{tabular}{llll}
\hline Organisms isolated & Aerobic & Anaerobic & Malthus \\
\hline Escherichia coli & 22 & 18 & 22 \\
Pseudomonas aeruginosa & 19 & 21 & 22 \\
Other Gram-negative & & & \\
facultative organisms & 16 & 14 & 13 \\
Staphylococcus aureus & 8 & 5 & 6 \\
Streptococci (10 species) & 18 & 16 & 18 \\
Clostridium spp and & 9 & 12 & 7 \\
Bacteroides spp & 0 & 1 & 1 \\
Candida albicans & 92 & 87 & 89 \\
\hline Total significant isolates & &
\end{tabular}

clinically significant were grown from at least one of the three bottles inoculated (Table 1). In total, organisms considered to be clinically significant were isolated from 82 aerobic, 78 anaerobic and 71 Malthus bottles. The differences among these numbers were not significant by the $\chi^{2}$ test $(p>0.05)$.

Organisms which were assessed clinically to be contaminants were Staph epidermidis, micrococci, diphtheroids, Bacillus sp and a Gram-negative nonfermenting bacillus. The number of aerobic, anaerobic and Malthus bottles containing contaminants only were 32,20 and 16 respectively. When assessed by the $\chi^{2}$ test there were significant differences between the contamination rates in the aerobic and Malthus bottles $(p<0.02)$ only. Of the 16 contaminated Malthus bottles 10 were not subcultured before detection of the contaminant and one of the remaining six was contaminated with the same organism as was one of the other bottles in the set.

ORGANISMS ISOLATED

More than one species or strain was isolated from a single bottle on several occasions; hence the number of isolates was greater than the number of bottles positive (Table 2). The numbers of significant isolates from aerobic, anaerobic and Malthus bottles were 92,87 and 89 respectively.

\section{DETECTION OF POSITIVE CULTURES BY THE MALTHUS SYSTEM}

Of the 87 Malthus bottles in which organisms were detected (71 significant, 16 contaminated) eight were detected as positive by subcultures made before the bottles were connected to the system; changes in conductivity indicating growth in these bottles were not subsequently noted. One hundred and eightyfour bottles in the Malthus system required subculture because the cultures were taken at night and incubated before being connected to the apparatus. The electrodes in two bottles failed so that these could not be assessed although organisms were isolated from them on subculture. Of the remaining 77 bottles in which organisms were detected, growth was indicated by changes in conductivity in 76 bottles. In one bottle a strain of Staph epidermidis was not detected by the Malthus system.

Instability of some electrodes causes significant difficulty in assessing whether changes in conductivity were or were not due to growth of microorganisms and resulted in unnecessary subculture of 175 bottles $(26.9 \%)$. These were regarded as falsepositive results.

\section{TIME TO DETECTION OF SIGNIFICANT POSITIVE CULTURES}

The same significant organism was detected by the Malthus system and by either (or by both) of the conventional bottles in 55 cultures so comparison can be made of the time taken to the first indication of growth (Table 3 ). When the Malthus system was assessed between 09.00 and $17.30 \mathrm{~h}, 15(27 \cdot 3 \%)$ of the 55 positive cultures were detected earlier by the Malthus system, $14(25.5 \%)$ being at least eight hours earlier. The conventional system detected nine $(16.4 \%)$ of the 55 positive cultures earlier than did the Malthus system, with three $(5.5 \%)$ being more than eight hours earlier.

Table 3 Relative detection times of the Malthus and conventional blood culture methods for 55 positive cultures

\begin{tabular}{|c|c|c|c|c|}
\hline \multirow[t]{3}{*}{ Relative detection time(h) } & \multicolumn{4}{|c|}{ Number of positive cultures detected earlier by } \\
\hline & \multicolumn{2}{|l|}{ Malthus system } & \multicolumn{2}{|c|}{ Conventional system } \\
\hline & $\begin{array}{l}\text { Actual Malthus } \\
\text { detection time }\end{array}$ & $\begin{array}{l}09.00-17.30 \mathrm{~h} \\
\text { Malthus reading }\end{array}$ & $\begin{array}{l}\text { Actual Malthus } \\
\text { detection time }\end{array}$ & $\begin{array}{l}09.00-17.30 \mathrm{~h} \\
\text { Malthus reading }\end{array}$ \\
\hline $\begin{array}{l}\text { Less than } 8 \\
8 \cdot 5-16 \\
16 \cdot 5-24 \\
\text { Greater than } 24\end{array}$ & $\begin{array}{r}21 \\
10 \\
6 \\
9\end{array}$ & $\begin{array}{l}1 \\
3 \\
4 \\
7 \\
\end{array}$ & $\begin{array}{l}4 \\
0 \\
0 \\
0\end{array}$ & $\begin{array}{l}6 \\
0 \\
3 \\
0\end{array}$ \\
\hline
\end{tabular}


The organisms isolated from cultures detected more than eight hours earlier by the Malthus system were 10 strains of Pseudomonas aeruginosa, two of Salmonella typhimurium, one of Streptococcus faecium, and one of Capnocytophaga ochracea. Three cultures containing Staphylococcus aureus were detected more than eight hours earlier by the conventional system.

Since the Malthus system scans the bottles automatically every $30 \mathrm{~min}$ it can detect positive cultures at any time. If the detection time recorded by the Malthus equipment is compared with the detection time observed by the conventional system, 46 $(83.6 \%)$ of the 55 positive cultures were detected earlier by the Malthus system, $25(45.5 \%)$ being more than eight hours earlier. Four $(7 \cdot 3 \%)$ cultures were detected earlier by the conventional system but all less than eight hours earlier than the Malthus system.

\section{Discussion}

In this hospital all immunosuppressed patients with suspected bacteraemia are treated empirically with broad spectrum antibiotics before laboratory results are available because of the associated high mortality if they are not treated. The extent to which the laboratory findings may influence the treatment of bacteraemic patients is likely to increase with the speed of detection of organisms in blood cultures. In our hands, the Malthus system detected microorganisms in blood cultures faster than the conventional system in most instances. The extent to which such early information is useful will depend largely on working practice in the laboratory as the times at which positive cultures were detected by the Malthus system had been spread evenly throughout the day and night. Even if the system were used only between 09.00 and $17.30 \mathrm{~h}$, a quarter of the positive cultures would have been detected more than eight hours earlier by the Malthus system while only $5.5 \%$ would have been detected more than eight hours earlier by the conventional system. The earlier results compare favourably with those of the Bactec radiometric system, with which earlier detection than that obtained by conventional methods has been reported in most studies. ${ }^{8}$ The potential of the Malthus system for early detection is derived from the automatic reading every $30 \mathrm{~min}$ in the absence of laboratory staff. Furthermore, software now available can automatically signal the detection of positive cultures to a geographically remote source. In this study, conductivity traces were visually examined for indications of microbial growth. Although the magnitude of changes in conductivity varied considerably among species or organisms there was generally little difficulty in detecting growth. The only trace which failed to indicate bacterial growth (a contaminating Staph epidermidis), was difficult to interpret because of electrode instability. Reliability of the system depends to a great extent on stability of background conductivity. Adequate stability appears to have been achieved in later versions of the apparatus now under test.

The organisms detected significantly earlier by the Malthus system were mostly strains of Ps aeruginosa. There is no evidence that the Malthus system is particularly sensitive in detecting these organisms so there may be a deficiency in the conventional methods used. It has been suggested that numbers of Ps aeruginosa in excess of $10^{7}$ per $\mathrm{ml}$ are necessary before turbidity is observed in conventional blood culture bottles, ${ }^{9}$ and the results of the first-day blind subcultures may not be seen until after a further day's incubation. The strains of Staph aureus found earlier by the conventional system were detected in first-day blind subcultures when these were examined in the afternoon of the same day. These cultures were not detected by the Malthus system until later the same day.

A fully automated blood culture screening apparatus offers considerable savings in time and materials required for visual inspection and blind subculturing of bottles. These savings would be less however if the specificity of the automated system were not high. The $26.9 \%$ false positive rate in this study of the Malthus system was caused largely by unacceptable instability of the electrodes used at that time. Recent modifications have resulted in electrodes which are proving to be much more stable so that the falsepositive rate should be considerably less in the future. Anhalt ${ }^{8}$ quoted false-positive rates in studies of the Bactec of between $0 \%$ and $43 \%$. An additional need for subculture in the Malthus system, but not in radiometric systems, relates to the difficulty of ensuring that blood cultures are promptly connected to the apparatus. If prompt connection cannot be achieved, bottles need to be routinely subcultured at the time they are connected to the machine because in the Malthus system stationary phase cultures may not result in any signal that indicates microbial growth. Furthermore, turbidity of the culture medium caused by bacterial growth may not be obvious due to interference from erythrocytes partially lysed by the saponin that is added to the Malthus bottles.

Fewer Malthus than conventional bottles were contaminated in this study. The differences probably relate largely to the frequency of subculture of the bottles because the aerobic bottles were subcultured more often than the anaerobic bottles which in turn were subcultured more often than the Malthus bottles. The Malthus system of examination is non- 
invasive so that contaminants introduced in the laboratory should be rare. This advantage would be less, however, if bottles were often subcultured because of false positive signals or because bottles were not promptly attached to the apparatus. Organisms in two thirds of the contaminated Malthus bottles were probably introduced when the blood sample was taken, because either the bottles had not been subcultured before the contaminants were detected or because the contaminant was also present in another bottle in the same set.

This trial of the prototype Malthus system has shown that there could be considerable advantages in using the apparatus for continuous monitoring of blood cultures as regards the early detection of bacteraemia as well as economy of time and materials otherwise needed for visual examination and blind subculture of bottles. It is not certain that the results of this study are typical of the performance of the apparatus operated in a routine rather than a research environment and with specimens from patients having a wider variety of conditions and a wider range of infecting micro-organisms. Consequently a trial of a fully automated Malthus system operated in the routine microbiology laboratory is now in progress.

We are grateful to Professor FG Hayhoe and Dr DB Evans and their colleagues for collaborating in this trial, to the staff of the Cambridge Clinical Microbiology and Public Health Laboratory for their technical co-operation, to Malthus Instruments Ltd for the loan of equipment, to Mr DA Kennedy for advice and administrative assistance, and to Miss Ann Moore for preparing the manuscript. This work was supported by a grant from the Department of Health and Social Security.

\section{References}

${ }^{1}$ Ur A, Brown DFJ. Impedance monitoring of bacterial activity. $J$ Med Microbiol 1975;8:19-28.

${ }^{2}$ Cady P. Rapid automated bacterial identification by impedance measurement. In: Heden CG, Illeni T, eds. New approaches to the identification of micro-organisms. London: John Wiley, 1975: 73-9.

${ }^{3}$ Hadley WK, Kazinka W. Comparison of impedance measurements and standard laboratory procedures for detection of microorganisms in blood cultures. Abstracts, Annual MeetingAmerican Society for Microbiology 1976:M281.

${ }^{4}$ Khan W, Friedman G, Rodriguez W, Controni G, Ross S. Rapid detection of bacteria in blood and spinal fluids in children by electrical impedance method. Abtracts Annual MeetingAmerican Society for Microbiology 1976:C70.

${ }^{5}$ Richards JCS, Jason AC, Hobbs G, Gibson DM, Christie RH. Electronic measurement of bacterial growth. J Phys (Sect E) 1978;2:560-8.

${ }^{6}$ Holdeman LV, Cato EP, Moore WEC, eds. Anaerobe laboratory manual. Blacksburg, Virginia: VPI Anaerobe Laboratory, 1977.

' Cowan ST, Steele KJ. Manual for the identification of medical bacteria. 2nd ed. London: Cambridge University Press, 1974.

- Anhalt JP. New or experimental approaches to detection of bacteraemia. In: Washington JA, ed. The detection of septicemia West Palm Beach, Fla: CRC Press Inc, 1978;109-44.

${ }^{9}$ Braunstein H, Tomasulo M. A quantitative study of the multiplication of Pseudomonas aeruginosa in vented and unvented bloodculture bottles. Am J Clin Pathol 1976;66:80-90.

Requests for reprints to: Dr DFJ Brown, Public Health Laboratory, Level 6, Addenbrooke's Hospital, Hills Road, Cambridge CB2 2QW, England. 